\title{
Symmetry Operators
}

\section{for the Fokker-Plank-Kolmogorov}

\section{Equation with Nonlocal Quadratic Nonlinearity ${ }^{\star}$}

\author{
Alexander V. SHAPOVALOV ${ }^{\dagger}$, Roman O. REZAEV ${ }^{\ddagger}$ and Andrey Yu. TRIFONOV $\ddagger$ \\ $\dagger$ Theoretical Physics Department, Tomsk State University, \\ 36 Lenin Ave., 660050, Tomsk, Russia \\ E-mail: shpv@phys.tsu.ru \\ $\ddagger$ Laboratory of Mathematical Physics, Mathematical Physics Department, \\ Tomsk Polytechnical University, 30 Lenin Ave., 660034, Tomsk, Russia \\ E-mail: rezaev@tpu.ru,trifonov@phtd.tpu.edu.ru
}

Received October 11, 2006, in final form December 09, 2006; Published online January 05, 2007

Original article is available at http://www.emis.de/journals/SIGMA/2007/005/

\begin{abstract}
The Cauchy problem for the Fokker-Plank-Kolmogorov equation with a nonlocal nonlinear drift term is reduced to a similar problem for the correspondent linear equation. The relation between symmetry operators of the linear and nonlinear Fokker-PlankKolmogorov equations is considered. Illustrative examples of the one-dimensional symmetry operators are presented.
\end{abstract}

Key words: symmetry operators; Fokker-Plank-Kolmogorov equation; nonlinear partial differential equations

2000 Mathematics Subject Classification: 35Q58; 37J15

\section{Introduction}

By definition, symmetry operators leave invariant the solution set of an equation and allow to generate new solutions from the known ones (see, e.g. [1, 2]). The finding of symmetry operators is an important problem, but it rarely can be solved explicitly because the equations that determine symmetry operators are complicated and nonlinear. Therefore, special types of symmetry operators are of interest. The most affective approach is developed in the framework of the group analysis of differential equations $[3,4,5,6,7]$ where the Lie groups of symmetry operators are considered. The Lie group generators (related to symmetries) are obtained from the determining linear equations which can be solved in a regular way when the symmetries are differential operators. The symmetries of differential equations can be considered in the context of differential geometry [5, 8, 9].

The calculation of symmetries for integro-differential equations is a more complex problem because there are no general way of choosing an appropriate structure of symmetries. The finding of symmetry operators for a nonlocal equation is usually a hopeless task. Under these circumstances, examples of symmetry operators for a nonlinear equation with nonlocal terms are of mathematical interest.

In this work we consider an approach that can be used to obtain such examples for the Fokker-Planck-Kolmogorov equation (FPKE) of special form with a quadratic nonlocal nonli-

`This paper is a contribution to the Vadim Kuznetsov Memorial Issue "Integrable Systems and Related Topics". The full collection is available at http://www.emis.de/journals/SIGMA/kuznetsov.html 
nearity

$$
\left\{-\partial_{t}+\varepsilon \Delta+\partial_{\vec{x}}\left(\vec{V}(\vec{x}, t)+\varkappa \int_{\mathbb{R}^{n}} \vec{W}(\vec{x}, \vec{y}, t) u(\vec{y}, t) d \vec{y}\right)\right\} u(\vec{x}, t)=0,
$$

where

$$
\vec{V}(\vec{x}, t)=K_{1} \vec{x}, \quad \vec{W}(\vec{x}, \vec{y}, t)=K_{2} \vec{x}+K_{3} \vec{y} .
$$

Here, $t \in \mathbb{R}^{1}, \vec{x}=\left(x_{1}, \ldots, x_{n}\right)^{\top} \in \mathbb{R}^{n}, \vec{y}=\left(y_{1}, \ldots, y_{n}\right)^{\top} \in \mathbb{R}^{n}$ are independent variables; $\left(x_{1}, \ldots, x_{n}\right)^{\top}$ means a transpose to a vector or a matrix; $d \vec{x}=d x_{1} \cdots d x_{n}$; the dependent variable $u(\vec{x}, t)$ is a real smooth function decreasing as $\|\vec{x}\| \rightarrow \infty ; K_{1}, K_{2}, K_{3}$ are arbitrary constant matrices of order $n \times n ; \varepsilon$ and $\varkappa$ are real parameters; $\partial_{t}=\partial / \partial t ; \partial_{\vec{x}}=\partial / \partial \vec{x}$ is a gradient operator with respect to $\vec{x} ; \Delta=\partial_{\vec{x}} \partial_{\vec{x}}=\sum_{i=1}^{n} \partial^{2} / \partial x_{i}^{2}$ is a Laplace operator.

The operator of equation (1.1) is quadratic in independent variables and in derivatives and it has a nonlocal quadratic nonlinear term.

This equation serves as a simple example of a class of "near-linear" nonlocal equations [10], such that they are nonlinear but the integrability problem for them can be reduced to seeking a solution of appropriate linear equations. Nonlinear equations of such type regularly depend on the nonlinearity parameter and they possess solutions which go into solutions of the linear equation as the nonlinearity parameter tends to zero.

Equation (1.1) arises in mathematical problems and it can be used in physical applications. In particular, the FPKE (1.1), (1.2) describes the leading term of the asymptotic solutions constructed in [11] in the framework of the formalism of semiclassical asymptotics [12, 13] for equation (1.1), in which $\vec{V}(\vec{x}, t)$ and $\vec{W}(\vec{x}, t)$ are real vector functions of general form.

The semiclassical approximation is widely used in nonlinear mathematical physics, providing a possibility of constructing explicit asymptotic solutions for mathematical physics equations coefficients of which can be arbitrary smooth functions and derivatives of dependent variables are assumed small. Most of the equations solved by semiclassical methods are not exactly integrable. For these equations, semiclassical methods offer a unique opportunity to investigate them analytically.

A method of semiclassical asymptotics based on the formalism of the Maslov complex germ $[12,13,14,15,16]$ has been developed for a many-dimensional nonstationary Hartree type equation with nonlocal nonlinearity in a class of functions localized in a neighborhood of some phase curve $[17,18,19,20]$. This class of functions has been called the class of trajectory-concentrated functions (TCF). The Hartree type equation whose operator is quadratic in independent variables and derivatives provides another example of the class of "near-linear" nonlinear equations similar to the FPKE (1.1).

The symmetry analysis area may be augmented by the study of the symmetry features of the semiclassical approximation as the semiclassical methods are hoped to result in a new kind of symmetries for mathematical physics equations. The group properties of the semiclassical approximation were considered in quantum mechanics and in some models of the quantum field theory [21]. The semiclassical method for solving the Cauchy problem in the class of TCF's has been developed for the Hartree type equation [17, 18, 19, 20] and for the one-dimensional FPKE $[11,22,23]$. For the Hatree type equation, symmetry operators have been found in the TCF class.

A nonlinear FPKE was used to analyze stochastic processes in various physical phenomena. In this connection, the following works where the Fokker-Plank-Kolmogorov equation with the nonlinear drift term similar to that in (1.1) was considered deserve mention (see also [10] and references therein). M. Shiino and K. Yoshida studied noise effects and phase transitions effects involving chaos-nonchaos bifurcations [24] in the framework of nonlinear Fokker-Plank 
equations. These equations are shown to exhibit the property of $H$ theorem with a Lyapunov functional that takes the form of free energy involving generalized entropies of Tsallis [25]. In [26] the stochastic resonance phenomenon is discussed; in [27] binary branching and dying processes were studied. The evolution of quantum systems was described by means of nonlinear FPKE's [28] where the nonlinearity reflects the quantum constraints imposed by the Bose and Fermi statistics.

The paper is organized as follows. In Section 2 the nonlinear FPKE is presented with necessary notations and definitions. The Cauchy problem for the nonlinear FPKE is reduced to a similar problem for the corresponding linear FPKE in the class of functions decreasing at infinity via the Cauchy problem for the first moment vector of a solution of the nonlinear FPKE. With the help of the Cauchy problem solution we construct a nonlinear evolution operator and the corresponding left inverse operator in explicit form for the nonlinear FPKE.

In Section 3 a general class of nonlinear symmetry operators is considered for the nonlinear FPKE. The symmetry operators are introduced in different ways, in particular using the evolution operator and the left inverse operator. Examples of one-dimensional symmetry operators are given in explicit form as an illustration. In Conclusion the results are discussed in the framework of symmetry analysis.

\section{The Cauchy problem and the evolution operator}

In our consideration, the key part is played by the Cauchy problem for the FPKE (1.1), (1.2) in the class of functions $u(\vec{x}, t)$ decreasing as $\|\vec{x}\| \rightarrow \infty$ at every point of time $t \geqslant 0$. To be specific, we assume that $u(\vec{x}, t)$ belongs to the Schwartz space $\mathcal{S}$ in the variable $\vec{x} \in \mathbb{R}^{n}$ and regularly depends on $t$, i.e. $u(\vec{x}, t)$ is expanded as a power series in $t$ about $t=0$. Obviously, equation (1.1) can be written in the form of the balance equation

$$
\partial_{t} u(\vec{x}, t)=\partial_{\vec{x}} \vec{B}(\vec{x}, t, u),
$$

where

$$
\vec{B}(\vec{x}, t, u)=\varepsilon \partial_{\vec{x}} u(\vec{x}, t)+\vec{V}(\vec{x}, t) u(\vec{x}, t)+\varkappa \int_{\mathbb{R}^{n}} \vec{W}(\vec{x}, \vec{y}, t) u(\vec{y}, t) d \vec{y} u(\vec{x}, t) .
$$

Then according to the divergence theorem, we obtain that the integral $\int_{\mathbb{R}^{n}} u(\vec{x}, t) d \vec{x}$ conserves in time $t$ for every solution $u(\vec{x}, t)$ of equation (1.1). Therefore, taking the initial function $u(\vec{x}, 0)=\gamma(\vec{x})$ to be normalized, $\int_{\mathbb{R}^{n}} \gamma(\vec{x}) d \vec{x}=1$, we can assume

$$
\int_{\mathbb{R}^{n}} u(\vec{x}, t) d \vec{x}=1, \quad t \geqslant 0,
$$

without loss of generality. We do not pay special attention to the positive definiteness of solutions of the FPKE (1.1), leaving this requirement for specific examples (see [29] for details).

Let us write equations (1.1), (1.2) in equivalent form

$$
\left\{-\partial_{t}+\widehat{H}_{\mathrm{nl}}\left(\vec{x}, t ; \vec{X}_{u}(t)\right)\right\} u(\vec{x}, t)=0,
$$

where the operator $\widehat{H}_{\mathrm{nl}}$ reads

$$
\widehat{H}_{\mathrm{nl}}\left(\vec{x}, t ; \vec{X}_{u}(t)\right)=\varepsilon \Delta+\partial_{\vec{x}}\left(\Lambda \vec{x}+\varkappa K_{3} \vec{X}_{u}(t)\right),
$$

the matrix $\Lambda$ is

$$
\Lambda=K_{1}+\varkappa K_{2},
$$


and the vector

$$
\vec{X}_{u}(t)=\int_{\mathbb{R}^{n}} \vec{x} u(\vec{x}, t) d \vec{x}
$$

is the first moment of the function $u(\vec{x}, t)$. With the obvious notation $\dot{\vec{X}}_{u}(t)=d \vec{X}_{u}(t) / d t$, we obtain immediately from $(2.2)-(2.4)$, and $(2.1)$

$$
\dot{\vec{X}}_{u}(t)=-\left(\Lambda+\varkappa K_{3}\right) \vec{X}_{u}(t) .
$$

Equation (2.5) can be considered the first equation of the Einstein-Ehrenfest system (EES) that describes the evolution of the moments and centered high-order moments of a solution $u(\vec{x}, t)$ of the FPKE (1.1) with the vector functions $\vec{V}(\vec{x}, t)$ and $\vec{W}(\vec{x}, t)$ of general form. The total EES for moments of all orders was derived in constructing approximate semiclassical solutions for a one-dimensional FPKE in [11].

\subsection{Solution of the Cauchy problem}

Let us set the Cauchy problem for equation (2.2):

$$
u(\vec{x}, 0)=\gamma(\vec{x}), \quad \gamma(\vec{x}) \in \mathcal{S}, \quad \int_{\mathbb{R}^{n}} \gamma(\vec{x}) d \vec{x}=1 .
$$

Then we have the induced Cauchy problem for the vector $\vec{X}_{u}(t)$

$$
\vec{X}_{u}(0)=\vec{X}_{\gamma}=\int_{\mathbb{R}^{n}} \vec{x} \gamma(\vec{x}) d \vec{x}
$$

determined by (2.5).

The nonlinear Cauchy problem (2.2), (2.6) is reduced to a linear one as follows. For a given initial function $\gamma(\vec{x})(2.6)$, we can seek a solution of the Cauchy problem (2.5), (2.7) independently of the solution of equation (2.2) and obtain the vector $\vec{X}_{u}(t)$ having the form of (2.4) due to the uniqueness of the Cauchy problem solution. Let us introduce a function $w(\vec{x}, t)$ by the equality

$$
u(\vec{x}, t)=w\left(\vec{x}-\vec{X}_{u}(t), t\right) .
$$

By substitution of $(2.8)$ in $(2.2)$ we obtain for the function $w(\vec{x}, t)$ a linear equation:

$$
\begin{aligned}
& -\partial_{t} w(\vec{x}, t)+\widehat{L} w(\vec{x}, t)=0, \\
& \widehat{L}=\varepsilon \Delta+\partial_{\vec{x}} \Lambda \vec{x} .
\end{aligned}
$$

From (2.6) and (2.8) we have

$$
w(\vec{x}, 0)=\tilde{\gamma}(\vec{x})=\gamma\left(\vec{x}+\vec{X}_{\gamma}\right)
$$

and

$$
\int_{\mathbb{R}^{n}} \tilde{\gamma}(\vec{x}) d \vec{x}=1 .
$$

Equation (2.7) results in

$$
\int_{\mathbb{R}^{n}} \vec{x} \tilde{\gamma}(\vec{x}) d \vec{x}=0,
$$


i.e. the function $\tilde{\gamma}(\vec{x})$ is centered. Obviously, the integral $\int_{\mathbb{R}^{n}} w(\vec{x}, t) d \vec{x}$ conserves in time $t$ for any solution $w(\vec{x}, t)$ of equation (2.9); then from (2.12) we have

$$
\int_{\mathbb{R}^{n}} w(\vec{y}, t) d \vec{y}=1
$$

Therefore, the nonlinear Cauchy problem (2.2), (2.3), (2.6) can be solved as follows. First, for a given initial function $\gamma(\vec{x})(2.6)$ we solve the linear Cauchy problem (2.9), (2.10), (2.11) with the initial function $\tilde{\gamma}(\vec{x})$ normalized by condition (2.12) and centered by (2.13). Second, we find the vector $\vec{X}_{u}(t)$ by solving the Cauchy problem $(2.5),(2.7)$. Then, the solution of the nonlinear Cauchy problem (2.2), (2.3), (2.6) is given by (2.8).

Equation (2.9) is known (see, e.g., [2]) to have a solution in the form of a Gaussian wave packet:

$$
w(\vec{x}, t)=\sqrt{\frac{\operatorname{det} Q(t)}{(2 \pi \varepsilon)^{n}}} \exp \left[-\frac{1}{2 \varepsilon} \vec{x}^{\top} Q(t) \vec{x}\right],
$$

where $Q(t)$ is a symmetric positive-definite matrix of order $n \times n$. Substituting (2.14) in (2.9), we obtain

$$
\begin{aligned}
& \vec{x}^{\top} \dot{Q}(t) \vec{x}+2 \vec{x}^{\top}(Q(t))^{2} \vec{x}-\vec{x}^{\top} \Lambda^{\top} Q(t) \vec{x}-\vec{x}^{\top} Q(t) \Lambda \vec{x}-\varepsilon \frac{d}{d t} \log \operatorname{det} Q(t) \\
& \quad+2 \varepsilon \operatorname{Tr}(-Q(t)+\Lambda)=0 .
\end{aligned}
$$

Here $\operatorname{Tr} \Lambda$ is the trace of the matrix $\Lambda$. Equating the coefficients of equal powers of $\vec{x}$, we have

$$
\begin{aligned}
& \dot{Q}(t)+2(Q(t))^{2}-\Lambda^{\top} Q(t)-Q(t) \Lambda=0, \\
& -\frac{d}{d t} \log \operatorname{det} Q(t)+2 \operatorname{Tr}(-Q(t)+\Lambda)=0 .
\end{aligned}
$$

Let us take $Q(t)$ in the form

$$
Q(t)=B(t)(C(t))^{-1},
$$

where $B(t)$ and $C(t)$ are matrices of order $n \times n$. On substitution of (2.16) in (2.15) we can write

$$
\begin{array}{llrl}
\dot{B}(t) & =\Lambda^{\top} B(t), & B(0) & =B_{0}, \\
\dot{C}(t) & =2 B(t)-\Lambda C(t), & C(0) & =C_{0},
\end{array}
$$

where $B_{0}$ and $C_{0}$ are arbitrary constant matrices of $n \times n$ order. We call equations (2.17) a system in variations in matrix form.

For the one-dimensional case, the linear equation (2.9) takes the form

$$
\left\{-\partial_{t}+\varepsilon \partial_{x}^{2}+\partial_{x} \Lambda x\right\} w(x, t)=0,
$$

where $\partial_{x}=\partial / \partial x$.

The solution (2.14) of equation (2.18) reads

$$
w(x, t)=\sqrt{\frac{B(t)}{2 \pi \varepsilon C(t)}} \exp \left[-\frac{B(t)}{2 \varepsilon C(t)} x^{2}\right],
$$

where $B(t)$ and $C(t)$ are a solution of the system in variations (2.17) in the one-dimensional case. For $t=0$ we have

$$
w(x, 0)=\widetilde{\gamma}(x)=\sqrt{\frac{B_{0}}{2 \pi \varepsilon C_{0}}} \exp \left[-\frac{B_{0}}{2 \varepsilon C_{0}} x^{2}\right] .
$$


Notice that the function $\widetilde{\gamma}(x)(2.19)$ is normalized and centered:

$$
\int_{-\infty}^{+\infty} \tilde{\gamma}(x) d x=1, \quad \int_{-\infty}^{+\infty} x \tilde{\gamma}(x) d x=0 .
$$

Then the function

$$
u(x, t)=w\left(x-X_{u}(t), t\right)=\sqrt{\frac{B(t)}{2 \pi \varepsilon C(t)}} \exp \left[-\frac{B(t)}{2 \varepsilon C(t)}\left(x-X_{u}(t)\right)^{2}\right]
$$

will be a solution of the nonlinear equation

$$
\left\{-\partial_{t}+\varepsilon \partial_{x}^{2}+\partial_{x} \Lambda x+\varkappa K_{3} X_{u}(t) \partial_{x}\right\} u(x, t)=0,
$$

with the initial condition

$$
u(x, 0)=\gamma(x)=\tilde{\gamma}\left(x-X_{\gamma}\right)=\sqrt{\frac{B_{0}}{2 \pi \varepsilon C_{0}}} \exp \left[-\frac{B_{0}}{2 \varepsilon C_{0}}\left(x-X_{\gamma}\right)^{2}\right] .
$$

The vector $X_{u}(t)=\int_{-\infty}^{+\infty} x u(x, t) d x$ in equation (2.21) satisfies the condition

$$
\dot{X}_{u}(t)=-\left(\Lambda+\varkappa K_{3}\right) X_{u}(t), \quad X_{u}(0)=X_{\gamma} .
$$

\subsection{The evolution operator for a nonlinear FPKE}

Let us rewrite the solution of the above nonlinear Cauchy problem in terms of the corresponding nonlinear evolution operator.

Let $G_{\text {lin }}(t, s, \vec{x}, \vec{y})$ be the Green function of the linear equation (2.9), i.e.

$$
w(\vec{x}, t)=\int_{\mathbb{R}^{n}} G_{\operatorname{lin}}(t, s, \vec{x}, \vec{y}) \tilde{\gamma}(\vec{y}) d \vec{y} .
$$

Substituting $\vec{x}$ for $\vec{x}-\vec{X}_{u}(t)$, we find

$$
w\left(\vec{x}-\vec{X}_{u}(t), t\right)=\int_{\mathbb{R}^{n}} G_{\operatorname{lin}}\left(t, s, \vec{x}-\vec{X}_{u}(t), \vec{y}\right) \gamma\left(\vec{y}+\vec{X}_{\gamma}\right) d \vec{y} .
$$

According to (2.8) and (2.11), the function

$$
u(\vec{x}, t)=\int_{\mathbb{R}^{n}} G_{\mathrm{nl}}(t, s, \vec{x}, \vec{y}, \gamma) \gamma(\vec{y}) d \vec{y}=\int_{\mathbb{R}^{n}} G_{\operatorname{lin}}\left(t, s, \vec{x}-\vec{X}_{u}(t), \vec{y}-\vec{X}_{\gamma}\right) \gamma(\vec{y}) d \vec{y}
$$

is a solution of the nonlinear equation (2.2), (2.3) with the initial condition (2.6). Therefore,

$$
G_{\mathrm{nl}}(t, s, \vec{x}, \vec{y}, \gamma)=G_{\operatorname{lin}}\left(t, s, \vec{x}-\vec{X}_{u}(t), \vec{y}-\vec{X}_{\gamma}\right)
$$

is the kernel of the evolution operator for the nonlinear equation (2.2). Here $\gamma(\vec{x})=u(\vec{x}, s)$ and the initial time $t=0$ is replaced by $t=s$.

Suppose that a solution of the system in variations (2.17) has the form

$$
B(t)=M_{1}(t, s) B_{0}, \quad C(t)=M_{2}(t, s) B_{0}+M_{3}(t, s) C_{0},
$$

where $M_{1}(t, s), M_{2}(t, s)$, and $M_{3}(t, s)$ are the matrix blocks of order $n \times n$ of the matriciant (evolution matrix) of the system in variations (2.17). These matrix blocks must satisfy the condition

$$
\dot{\mathrm{M}}=\mathrm{AM}, \quad \mathrm{M}(s)=\mathbb{I}_{2 n \times 2 n},
$$


where

$$
\mathrm{M}=\mathrm{M}(t, s)=\left(\begin{array}{cc}
M_{1}(t, s) & 0 \\
M_{2}(t, s) & M_{3}(t, s)
\end{array}\right), \quad \mathrm{A}=\left(\begin{array}{cc}
\Lambda^{\top} & 0 \\
2 \mathbb{I}_{n \times n} & -\Lambda
\end{array}\right) .
$$

Set the Cauchy problem for (2.26):

$$
B(s)=B_{0}^{\top}=B_{0}, \quad C(s)=C_{0}=0 .
$$

The Green's function of equation (2.9) is known and can be taken as (see, e.g., [2]):

$$
\begin{aligned}
G_{\operatorname{lin}}(t, s, \vec{x}, \vec{y})= & \frac{1}{\sqrt{(2 \pi \varepsilon)^{n} \operatorname{det}\left[M_{2}(t, s)\left(M_{1}(t, s)\right)^{-1}\right]}} \\
& \times \exp \left\{-\frac{1}{2 \varepsilon}\left(\vec{x}-M_{3}(t, s) \vec{y}\right)^{\top}\left[M_{1}(t, s)\left(M_{2}(t, s)\right)^{-1}\right]\left(\vec{x}-M_{3}(t, s) \vec{y}\right)\right\} .
\end{aligned}
$$

Then the nonlinear evolution $\widehat{U}(t, s, \cdot)$ operator $(2.24)$ reads

$$
\widehat{U}(t, s, \gamma)(\vec{x})=\int_{\mathbb{R}^{n}} G_{\mathrm{nl}}(t, s, \vec{x}, \vec{y}, \gamma) \gamma(\vec{y}) d \vec{y} .
$$

The left-inverse operator $\widehat{U}^{-1}(t, s, \cdot)$ for the operator $(2.28)$ is

$$
\widehat{U}^{-1}(t, s, u)(\vec{x})=\int_{\mathbb{R}^{n}} G_{\mathrm{nl}}^{-1}(t, s, \vec{x}, \vec{y}, u) u(\vec{x}, t) d \vec{x} .
$$

Here $G_{\mathrm{nl}}^{-1}(t, s, \vec{x}, \vec{y}, u)$ is the kernel of the left-inverse operator, which is obtained from (2.25) if we substitute $t$ for $s$ and $s$ for $t$. The explicit form of this function is

$$
\begin{aligned}
G_{\mathrm{nl}}^{-1}(t, s, \vec{x}, \vec{y}, u)= & \frac{1}{\sqrt{(2 \pi \varepsilon)^{n} \operatorname{det}\left[M_{2}(s, t)\left(M_{1}(s, t)\right)^{-1}\right]}} \\
& \times \exp \left\{-\frac{1}{2 \varepsilon}\left(\vec{x}-\vec{X}_{\gamma}-M_{3}(s, t)\left(\vec{y}-\vec{X}_{u}(t)\right)\right)^{\top}\left[M_{1}(s, t)\left(M_{2}(s, t)\right)^{-1}\right]\right. \\
& \left.\times\left(\vec{x}-\vec{X}_{\gamma}-M_{3}(s, t)\left(\vec{y}-\vec{X}_{u}(t)\right)\right)\right\} .
\end{aligned}
$$

The Green's function for the one-dimensional equation (2.18) is

$$
G_{\operatorname{lin}}(t, s, x, y)=\sqrt{\frac{M_{1}(t, s)}{2 \pi \varepsilon M_{2}(t, s)}} \exp \left[-\frac{1}{2 \varepsilon} \frac{M_{1}(t, s)}{M_{2}(t, s)}\left(x-M_{3}(t, s) y\right)^{2}\right],
$$

where $M_{1}(t, s), M_{2}(t, s), M_{3}(t, s)$ are solution of the system in variation $(2.27)$ in the onedimensional case.

The kernel of the evolution operator for the nonlinear equation (2.21) takes the form

$$
\begin{aligned}
G_{\mathrm{nl}}(t, s, x, y, \gamma) & =G_{\operatorname{lin}}\left(t, s, x-X_{u}(t), y-X_{\gamma}\right) \\
& =\sqrt{\frac{M_{1}(t, s)}{2 \pi \varepsilon M_{2}(t, s)}} \exp \left[-\frac{1}{2 \varepsilon} \frac{M_{1}(t, s)}{M_{2}(t, s)}\left(x-X_{u}(t)-M_{3}(t, s)\left(y-X_{\gamma}\right)\right)^{2}\right],
\end{aligned}
$$

where $X_{u}(t)$ satisfies equation (2.23). The evolution operator (2.28) with the kernel (2.30) is written as

$$
u(x, t)=\widehat{U}(t, s, \gamma)(x)=\int_{-\infty}^{+\infty} G_{\operatorname{lin}}\left(t, s, x-X_{u}(t), y-X_{\gamma}\right) \gamma(y) d y
$$

Here the function $u(x, t)$ having the form of (2.31) is a solution of equation (2.21). 
Notice that direct calculation of the action of the evolution operator (2.28) on the function $\gamma(y)$ having the form of (2.22) gives the function (2.20):

$$
\begin{aligned}
\widehat{U}(t, s, \gamma)(\vec{x})= & \int_{-\infty}^{+\infty} \sqrt{\frac{M_{1}(t, s)}{2 \pi \varepsilon M_{2}(t, s)}} \\
& \times \exp \left[-\frac{1}{2 \varepsilon} \frac{M_{1}(t, s)}{M_{2}(t, s)}\left(x-X_{u}(t)-M_{3}(t, s)\left(y-X_{\gamma}\right)\right)^{2}\right] \\
& \times \sqrt{\frac{B_{0}}{2 \pi \varepsilon C_{0}}} \exp \left[-\frac{B_{0}}{2 \varepsilon C_{0}}\left(y-X_{\gamma}\right)^{2}\right] d y \\
& =\sqrt{\frac{B(t)}{2 \pi \varepsilon C(t)}} \exp \left[-\frac{B(t)}{2 \varepsilon C(t)}\left(x-X_{u}(t)\right)^{2}\right] .
\end{aligned}
$$

Conversely, the action of the left-inverse operator (2.29) on the function (2.20) in the onedimensional case gives the function (2.22):

$$
\begin{aligned}
\widehat{U}^{-1}(t, s, u)(\vec{x})= & \int_{-\infty}^{+\infty} \sqrt{\frac{M_{1}(s, t)}{2 \pi \varepsilon M_{2}(s, t)}} \\
& \times \exp \left[-\frac{1}{2 \varepsilon} \frac{M_{1}(s, t)}{M_{2}(s, t)}\left(x-X_{\gamma}-M_{3}(s, t)\left(y-X_{u}(t)\right)\right)^{2}\right] \\
& \times \sqrt{\frac{B(t)}{2 \pi \varepsilon C(t)}} \exp \left[-\frac{B(t)}{2 \varepsilon C(t)}\left(y-X_{u}(t)\right)^{2}\right] d y \\
= & \sqrt{\frac{B_{0}}{2 \pi \varepsilon C_{0}}} \exp \left[-\frac{B_{0}}{2 \varepsilon C_{0}}\left(x-X_{\gamma}\right)^{2}\right] .
\end{aligned}
$$

Because the solution of the nonlinear equation (2.2) is reduced to seeking the solution of linear equation (2.9) in terms of the moment $\vec{X}_{u}(t)(2.5)$, the symmetry operators of these two equations are closely connected.

Equation (2.9) with the operator $\widehat{L}$ having the form of (2.10) is a special case of the linear evolution equation quadratic in derivatives $\partial_{\vec{x}}$ and independent variables $\vec{x}$. This equation is known to be integrated in explicit form (see, e.g., [2]) which in turn leads to integrability of the nonlinear FPKE (2.2), (2.3) according to (2.8) or (2.24). The basis of solutions and the Green's function for equation (2.9) can be constructed with the help of symmetry operators of special form following, for example, [2, 30, 31].

Consider the symmetry operators for (2.9) and (2.2).

\section{The symmetry operators}

The symmetry operators for equation (1.1) can be found in various ways following the general ideas of symmetry analysis $[2,3,4,5,6]$.

\subsection{The determining equation and intertwining}

Let us construct for a function $\gamma(\vec{x})$ of the space $\mathcal{S}$ the function $u(\vec{x}, t)$ of $(2.8)$ using the solutions of the Cauchy problems $(2.5),(2.7)$ for the vector $\vec{X}_{u}(t)$ and $(2.9),(2.11)$ for $w(\vec{x}, t)$.

Let us take an operator $\widehat{a}(\vec{x})$ acting in the space $\mathcal{S}$ as the initial operator for a time depending operator $\widehat{A}(\vec{x}, t)$ :

$$
\widehat{A}(\vec{x}, 0)=\widehat{a}(\vec{x}) .
$$


The function

$$
\gamma_{A}(\vec{x})=\frac{1}{\alpha_{A}} \widehat{a}(\vec{x}) \gamma(\vec{x}), \quad \alpha_{A}=\int_{\mathbb{R}^{n}} \widehat{a}(\vec{x}) \gamma(\vec{x}) d \vec{x} .
$$

determines the vector $\vec{X}_{\gamma_{A}}$ by formula $(2.7)$, where $\gamma(\vec{x})$ is replaced by $\gamma_{A}(\vec{x})$. Taking the vector $\vec{X}_{\gamma_{A}}$ as the initial condition for equation (2.5), we find the vector $\vec{X}_{u_{A}}(t)$.

Obviously, the operator $\widehat{A}(\vec{x}, t)$ determined by the conditions

$$
\begin{aligned}
& \left(-\partial_{t}+\widehat{H}_{\mathrm{nl}}\left(\vec{x}, t, \vec{X}_{u_{A}}(t)\right)\right) \widehat{A}(\vec{x}, t)=\widehat{B}(\vec{x}, t)\left(-\partial_{t}+\widehat{H}_{\mathrm{nl}}\left(\vec{x}, t, \vec{X}_{u}(t)\right)\right), \\
& \widehat{A}(\vec{x}, 0)=\widehat{a}(\vec{x})
\end{aligned}
$$

is a symmetry operator for equation $(2.2)$. Here $\widehat{B}(\vec{x}, t)$ is an operator such that $\widehat{B}(\vec{x}, t)(0)=0$. This operator plays the part of a Lagrangian multiplier and it is determined together with $\widehat{A}(\vec{x}, t)$.

Equation (3.2) is the determining equation for the symmetry operators of equation (2.2). In general, (3.2) is a nonlinear operator equation. But, given the initial function $\gamma(\vec{x})$ and the initial operator $\widehat{a}(\vec{x})$, we can find the vectors $\vec{X}_{u}(t)$ and $\vec{X}_{u_{A}}(t)$ without solving the equation (2.2). On substitution of these vectors in (3.2) the operators $\widehat{H}_{\mathrm{nl}}\left(\vec{x}, t, \vec{X}_{u}(t)\right)$ and $\widehat{H}_{\mathrm{nl}}\left(\vec{x}, t, \vec{X}_{u_{A}}(t)\right)$ become linear. Then we can assume that $\widehat{a}(\vec{x}), \widehat{A}(\vec{x}, t)$, and $\widehat{B}(\vec{x}, t)$ are linear operators in (3.2).

Notice that in the general case the operators $\widehat{A}$ and $\widehat{B}$ depend on $\vec{X}_{u}(t), \vec{X}_{u_{A}}(t)$, i.e.

$$
\widehat{A}=\widehat{A}\left(\vec{x}, t ; \vec{X}_{u}(t), \vec{X}_{u_{A}}(t)\right), \quad \widehat{B}=\widehat{B}\left(\vec{x}, t ; \vec{X}_{u}(t), \vec{X}_{u_{A}}(t)\right)
$$

If $\widehat{B}(\vec{x}, t)=\widehat{A}(\vec{x}, t)$ then $\widehat{A}(\vec{x}, t)$ is called the intertwining operator for the linear operators, satisfying the condition

$$
\begin{aligned}
& \left(-\partial_{t}+\widehat{H}_{\mathrm{nl}}\left(\vec{x}, t, \vec{X}_{u_{A}}(t)\right)\right) \widehat{A}(\vec{x}, t)=\widehat{A}(\vec{x}, t)\left(-\partial_{t}+\widehat{H}_{\mathrm{nl}}\left(\vec{x}, t, \vec{X}_{u}(t)\right)\right), \\
& \widehat{A}(\vec{x}, 0)=\widehat{a}(\vec{x}) .
\end{aligned}
$$

If the operator $\widehat{a}(\vec{x})$ is given in (3.2), (3.3) (or in (3.4), (3.5)), these conditions are the Cauchy problems determining the operator $\widehat{A}(\vec{x}, t)$.

Now, let $u(\vec{x}, t)$ be a solution of the Cauchy problem $(2.2),(2.3),(2.6)$, and the operator $\widehat{A}$ is determined by the solution of the Cauchy problem (3.2), (3.3) or (3.4), (3.5). Then we immediately obtain that the function

$$
u_{A}(\vec{x}, t)=\widehat{A}\left(\vec{x}, t ; \vec{X}_{u}(t), \vec{X}_{u_{A}}(t)\right) u(\vec{x}, t)
$$

is a solution of the Cauchy problem

$$
\begin{aligned}
& \partial_{t} u_{A}(\vec{x}, t)=\widehat{H}_{\mathrm{nl}}\left(\vec{x}, t, \vec{X}_{u_{A}}(t)\right) u_{A}(\vec{x}, t), \\
& u_{A}(\vec{x}, 0)=\gamma_{A}(\vec{x})=\frac{1}{\alpha_{A}} \widehat{a}(\vec{x}) \gamma(\vec{x}) .
\end{aligned}
$$

Therefore, the operator $\widehat{A}\left(\vec{x}, t ; \vec{X}_{u}(t), \vec{X}_{u_{A}}(t)\right)$ is a symmetry operator of the nonlinear FPKE (2.2), (2.3).

Notice that the operator $\widehat{A}\left(\vec{x}, t ; \vec{X}_{u}(t), \vec{X}_{u_{A}}(t)\right)$ in (3.6) is nonlinear due to the presence of the vectors $\vec{X}_{u}(t)$, and $\vec{X}_{u_{A}}(t)$. 


\subsection{Symmetry operators of nonlinear and linear FPKE}

We now deduce a relation connecting two solutions of the nonlinear FPKE (2.2), (2.3) using a symmetry operator of the linear equation (2.9), (2.10). This relation can be considered a symmetry operator for the nonlinear FPKE.

To this end consider equation (2.8) which connects the nonlinear Cauchy problem (2.6) with the linear Cauchy problem (2.11).

Let $\widehat{A}(\vec{x}, t)$ be a symmetry operator of the linear equation $(2.9)$. Then the function

$$
w_{A}(\vec{x}, t)=\frac{1}{\widetilde{\alpha}_{A}} \widehat{A}(\vec{x}, t) w(\vec{x}, t), \quad \widetilde{\alpha}_{A}=\int_{\mathbb{R}^{n}} \widehat{A}(\vec{x}, 0) w(\vec{x}, 0) d \vec{x},
$$

is another solution of the linear equation, which is determined by (2.9), (2.10). For $t=0$ we have

$$
w_{A}(\vec{x}, 0)=\frac{1}{\widetilde{\alpha}_{A}} \widehat{A}(\vec{x}, 0) \tilde{\gamma}(\vec{x}) \equiv \gamma_{A}(\vec{x})
$$

and

$$
\int_{\mathbb{R}^{n}} \gamma_{A}(\vec{x}) d \vec{x}=1
$$

which leads to normalization of the function $w_{A}(\vec{x}, t)$ :

$$
\int_{\mathbb{R}^{n}} w_{A}(\vec{x}, t) d \vec{x}=1
$$

On the other hand, the function $w_{A}(\vec{x}, t)$ is not centered for a symmetry operator $\widehat{A}(\vec{x}, t)$ of general form. In other words, for $t=0$ the vector

$$
\vec{\lambda}_{A}=\int_{\mathbb{R}^{n}} \vec{x} w_{A}(\vec{x}, 0) d \vec{x}=\int_{\mathbb{R}^{n}} \vec{x} \gamma_{A}(\vec{x}) d \vec{x}
$$

is nonzero.

To construct a solution of the nonlinear equation (2.2), which would correspond to the solution $w_{A}(\vec{x}, t)$ of the linear equation (2.9) with the use of relation $(2.8)$, the function $w_{A}(\vec{x}, t)$, being a solution of equation (2.9), should be centered.

We can immediately check that equation (2.9) is invariant under the change of variables $t^{\prime}=t, \vec{x}^{\prime}=\vec{x}-\vec{l}(t)$, where $\vec{l}(t)$ satisfies the condition $\dot{\vec{l}}(t)=-\Lambda \vec{l}(t)$.

Taking into account this property, let us introduce a vector

$$
\vec{l}_{A}(t)=\int_{\mathbb{R}^{n}} \vec{x} w_{A}(\vec{x}, t) d \vec{x}
$$

which satisfies the Cauchy problem

$$
\dot{\vec{l}}_{A}(t)=-\Lambda \vec{l}_{A}(t), \quad \vec{l}_{A}(0)=\vec{\lambda}_{A} .
$$

Then the function

$$
\tilde{w}_{A}(\vec{x}, t)=w_{A}\left(\vec{x}+\vec{l}_{A}(t), t\right)
$$

satisfies equation (2.9) and the initial condition

$$
\tilde{w}_{A}(\vec{x}, 0)=w_{A}\left(\vec{x}+\vec{\lambda}_{A}, 0\right)=\gamma_{A}\left(\vec{x}+\vec{\lambda}_{A}\right) .
$$

The function $\gamma_{A}\left(\vec{x}+\vec{\lambda}_{A}\right)$ is normalized and centered. The same is true for $\tilde{w}_{A}(\vec{x}, t)$. 
Following (2.8), we now construct a solution $v_{A}(\vec{x}, t)$ of the nonlinear FPKE $(2.2)$ related to $\tilde{w}_{A}(\vec{x}, t)$. Consider a vector $\vec{Y}(t)$ such that

$$
\dot{\vec{Y}}(t)=-\left(\Lambda+\varkappa K_{3}\right) \vec{Y}(t), \quad \vec{Y}(0)=\vec{\lambda}_{A} .
$$

Immediate check shows that the function

$$
v_{A}(\vec{x}, t)=\tilde{w}_{A}(\vec{x}-\vec{Y}(t), t)
$$

satisfies the equation

$$
\begin{aligned}
& \left\{-\partial_{t}+\varepsilon \Delta+\partial_{\vec{x}}\left(\Lambda \vec{x}+\varkappa K_{3} \vec{Y}(t)\right)\right\} v_{A}(\vec{x}, t)=0, \\
& v_{A}(\vec{x}, 0)=\widetilde{w}_{A}\left(\vec{x}-\vec{\lambda}_{A}, 0\right)=\gamma_{A}(\vec{x}) .
\end{aligned}
$$

Notice that

$$
\begin{aligned}
\vec{X}_{v_{A}}(t) & =\int_{\mathbb{R}^{n}} v_{A}(\vec{x}, t) \vec{x} d \vec{x}, \quad \vec{X}_{v_{A}}(0)=\int_{\mathbb{R}^{n}} \vec{x} \gamma_{A}(\vec{x}) d \vec{x}=\vec{\lambda}_{A}, \\
\dot{\vec{X}}_{v_{A}}(t) & =-\left(\Lambda+\varkappa K_{3}\right) \vec{X}_{v_{A}}(t),
\end{aligned}
$$

then

$$
\vec{Y}(t)=\vec{X}_{v_{A}}(t)
$$

Therefore, $v_{A}(\vec{x}, t)$ satisfies the nonlinear FPKE (2.2). The relation between the solutions $u(\vec{x}, t)$ and $v_{A}(\vec{x}, t)$ reads

$$
\begin{aligned}
v_{A}(\vec{x}, t) & =\tilde{w}_{A}(\vec{x}-\vec{Y}(t), t)=w_{A}\left(\vec{x}-\vec{Y}(t)+\vec{l}_{A}(t), t\right) \\
& =\frac{1}{\widetilde{\alpha}_{A}} \widehat{A}\left(\vec{x}-\vec{Y}(t)+\vec{l}_{A}(t), t\right) w\left(\vec{x}-\vec{Y}(t)+\vec{l}_{A}(t), t\right) \\
& =\frac{1}{\widetilde{\alpha}_{A}} \widehat{A}\left(\vec{x}-\vec{Y}(t)+\vec{l}_{A}(t), t\right) u\left(\vec{x}-\vec{Y}(t)+\vec{l}_{A}(t)+\vec{X}_{u}(t), t\right) .
\end{aligned}
$$

This equation determines a symmetry operator $\widehat{A}_{\mathrm{nl}}$ of the nonlinear FPKE (2.2):

$$
\begin{aligned}
u_{A}(\vec{x}, t) & =v_{A}(\vec{x}, t) \equiv \widehat{A}_{\mathrm{nl}}(\vec{x}, t) u(\vec{x}, t) \\
& =\frac{1}{\alpha_{A}} \widehat{A}\left(\vec{x}-\vec{Y}(t)+\vec{l}_{A}(t), t\right) u\left(\vec{x}-\vec{Y}(t)+\vec{l}_{A}(t)+\vec{X}_{u}(t), t\right) .
\end{aligned}
$$

\subsection{Symmetry operators in terms of an operator Cauchy problem}

Let us reformulate the construction of symmetry operators for the nonlinear FPKE (2.2), (2.3) in terms of an operator Cauchy problem.

Consider the nonlinear Cauchy problem (2.2), (2.6) associated with the Cauchy problem (2.5), (2.7) for the vector $\vec{X}_{u}(t)$ having the form of (2.4).

With an operator

$$
\widehat{a}(\vec{x}): \mathcal{S} \rightarrow \mathcal{S}
$$

acting on the initial function $\gamma(\vec{x}) \in \mathcal{S}$ of the Cauchy problem (2.6), we define a function $\gamma_{A}(\vec{x})$ of the form (3.1), which is taken as an initial condition for the Cauchy problem for a function $u_{A}(\vec{x}, t)$

$$
\left\{-\partial_{t}+\widehat{H}_{\mathrm{nl}}\left(\vec{x}, t, \vec{X}_{u_{A}}(t)\right)\right\} u_{A}(\vec{x}, t)=0,
$$


$u_{A}(\vec{x}, 0)=\gamma_{A}(\vec{x})$

where the vector $\vec{X}_{u_{A}}(t)$ is determined by the conditions

$$
\begin{aligned}
& \dot{\vec{X}}_{u_{A}}(t)=-\left(\Lambda+\varkappa K_{3}\right) \vec{X}_{u_{A}}(t), \\
& \vec{X}_{u_{A}}(0)=\vec{X}_{\gamma_{A}}, \quad \vec{X}_{\gamma_{A}}=\int_{\mathbb{R}^{n}} \vec{x} \gamma_{A}(\vec{x}) d \vec{x} .
\end{aligned}
$$

Notice that given the function $\gamma(\vec{x})$ and the operator $\widehat{a}(\vec{x})$, we can find $\vec{X}_{u_{A}}(t)$ not finding a solution of the FPKE (2.2).

We can immediately verify that the two functions

$$
\begin{aligned}
& w(\vec{x}, t)=u\left(\vec{x}+\vec{X}_{u}(t), t\right), \\
& w_{A}(\vec{x}, t)=u_{A}\left(\vec{x}+\vec{X}_{u_{A}}(t), t\right)
\end{aligned}
$$

are solutions of the linear equation (2.9) and the initial conditions are

$$
w(\vec{x}, 0)=\gamma\left(\vec{x}+\vec{X}_{\gamma}\right), \quad w_{A}(\vec{x}, 0)=\gamma_{A}\left(\vec{x}+X_{\gamma_{A}}\right) .
$$

Define a linear operator $\widehat{\bar{A}}(\vec{x}, t)$ by an operator equation

$$
\left[-\partial_{t}+\widehat{L}(\vec{x}, t), \widehat{\bar{A}}(\vec{x}, t)\right]=0
$$

with the initial condition

$$
\widehat{\bar{A}}(\vec{x}, 0)=\widehat{a}(\vec{x}),
$$

where $L(\vec{x}, t)$ is defined in $(2.10)$. It can be shown that

$$
\widehat{\bar{A}}(\vec{x}, t) w(\vec{x}, t)=\widehat{A}\left(\vec{x}+\vec{l}_{A}(t), t\right) w\left(\vec{x}+\vec{l}_{A}(t), t\right) .
$$

Due to the uniqueness of the Cauchy problem solution, we have

$$
w_{A}(\vec{x}, t)=\widehat{\bar{A}}(\vec{x}, t) w(\vec{x}, t) .
$$

In view of (3.9), (3.10) we have

$$
u_{A}\left(\vec{x}+\vec{X}_{u_{A}}(t), t\right)=\widehat{\bar{A}}(\vec{x}, t) u\left(\vec{x}+\vec{X}_{u}(t), t\right)
$$

or

$$
u_{A}(\vec{x}, t)=\widehat{\bar{A}}\left(\vec{x}-\vec{X}_{u_{A}}(t), t\right) u\left(\vec{x}-\vec{X}_{u_{A}}(t)+\vec{X}_{u}(t), t\right) .
$$

This relation defines a symmetry operator $\widehat{\bar{A}}_{\text {nl }}(\vec{x}, t)$ of the nonlinear FPKE $(2.2)$ :

$$
u_{A}(\vec{x}, t)=\widehat{\bar{A}}_{\mathrm{nl}}(\vec{x}, t) u(\vec{x}, t) .
$$




\subsection{Symmetry operators in terms of an evolution operator}

Using the evolution operator (2.28) and left-inverse operator (2.29), we can obtain symmetry operators for the nonlinear FPKE (2.2).

Let $\widehat{a}(\vec{x})$ be an operator (3.8) acting on an initial function $\gamma(\vec{x})$, and $u(\vec{x}, t)$ is the solution of the Cauchy problem (2.2), (2.6). Then the function

$$
u_{A}(\vec{x}, t)=\widehat{U}\left(t, s, \widehat{a} \widehat{U}^{-1}(t, s, u)\right)(\vec{x})
$$

is a solution of the nonlinear FPKE corresponding the initial function $\gamma_{A}(\vec{x})$ of the form (3.1). Equation (3.12) defines a symmetry operator $\widehat{\widetilde{A}}_{\mathrm{nl}}$ for the nonlinear FPKE (2.2):

$$
u_{A}(\vec{x}, t)=\widehat{\widetilde{A}}_{\mathrm{nl}}(\vec{x}, t) u(\vec{x}, t) .
$$

The one-dimensional case of (3.12) reads

$$
u_{A}(x, t)=\widehat{\widetilde{A}}_{\mathrm{nl}}(x, t) u(x, t)=\widehat{U}\left(t, s, \widehat{a} \widehat{U}^{-1}(t, s, u)\right)(x),
$$

where $\widehat{U}(t, s, \cdot)$ and $\widehat{U}^{-1}(t, s, \cdot)$ are determined by (2.28) and (2.29) in the one-dimensional case.

Consider an operator $\widehat{a}(x, t)$ of the form

$$
\begin{aligned}
& \widehat{a}(x, t)=M_{1}(t, s)\left(x-X_{u}(t)\right)+\left(\varepsilon M_{2}(t, s)+M_{3}(t, s)\right) \partial_{x}, \\
& \widehat{a}(x, s)=x-X_{\gamma}+\partial_{x}, \quad X_{\gamma}=X_{u}(s) .
\end{aligned}
$$

Here $M_{1}(t, s), M_{2}(t, s)$, and $M_{3}(t, s)$ are solutions of the system in variations $(2.27)$ in the one-dimensional case. Then for (3.14) we have

$$
\begin{aligned}
u_{A}(x, t)= & \lim _{\tau \rightarrow t} \widehat{U}\left(t, s,\left[\partial_{z}+z-X_{\gamma}\right] \widehat{U}^{-1}(\tau, s, u)\right)(x) \\
= & \lim _{\tau \rightarrow t} \int_{-\infty}^{+\infty} d y \int_{-\infty}^{+\infty} d z G_{\mathrm{nl}}\left(t, s, x, z, \gamma_{A}\right)\left[\partial_{z}+z-X_{\gamma}\right] G_{\mathrm{nl}}^{-1}(\tau, s, z, y, u) u(y, \tau) \\
= & \int_{-\infty}^{+\infty} d y \int_{-\infty}^{+\infty} d z \lim _{\tau \rightarrow t} \frac{1}{2 \pi \varepsilon} \sqrt{\frac{M_{1}(t, s) M_{1}(s, \tau)}{M_{2}(t, s) M_{2}(s, \tau)}} \\
& \times \exp \left\{-\frac{1}{2 \varepsilon}\left(x-X_{u_{A}}(t)-M_{3}(t, s)\left(z-X_{\gamma_{A}}\right)\right)^{2} \frac{M_{1}(t, s)}{M_{2}(t, s)}\right\}\left[\partial_{z}+z-X_{\gamma}\right] \\
& \times \exp \left\{-\frac{1}{2 \varepsilon}\left(z-X_{\gamma}-M_{3}(s, \tau)\left(y-X_{u}(\tau)\right)\right)^{2} \frac{M_{1}(s, \tau)}{M_{2}(s, \tau)}\right\} u(y, \tau) \\
= & {\left[M_{1}(t, s)\left(x-X_{u_{A}}(t)+M_{3}(t, s) X_{\gamma_{A}}\right)-X_{\gamma}+\left(\varepsilon M_{2}(t, s)+M_{3}(t, s)\right) \partial_{x}\right] } \\
& \times u\left(x+X_{u}(t)-X_{u_{A}}(t)+M_{3}(t, s)\left(X_{\gamma_{A}}-X_{\gamma}\right), t\right) \\
= & \widehat{a}\left(x+X_{u}(t)-X_{u_{A}}(t)+M_{3}(t, s)\left(X_{\gamma_{A}}-X_{\gamma}\right), t\right) u\left(x+X_{u}(t)-X_{u_{A}}(t)\right. \\
& \left.+M_{3}(t, s)\left(X_{\gamma_{A}}-X_{\gamma}\right), t\right) .
\end{aligned}
$$

Therefore, we have

$$
\begin{aligned}
u_{A}(x, t)= & \widehat{\widetilde{A}}_{\mathrm{nl}}(x, t) u(x, t)=\widehat{a}\left(x+X_{u}(t)-X_{u_{A}}(t)+M_{3}(t, s)\left(X_{\gamma_{A}}-X_{\gamma}\right), t\right) \\
& \times u\left(x+X_{u}(t)-X_{u_{A}}(t)+M_{3}(t, s)\left(X_{\gamma_{A}}-X_{\gamma}\right), t\right) .
\end{aligned}
$$

In calculating the symmetry operators we have used the following relations:

$$
M_{1}(t, s) M_{1}(s, \tau)=M_{1}(t, \tau), \quad M_{2}(t, \tau)=M_{1}(s, \tau) M_{2}(t, s)+M_{2}(s, \tau) M_{3}(t, s),
$$

which follow from (2.27) in the one-dimensional case. 
Equality (3.15) determines a symmetry operator in explicit form for equation (2.21). This symmetry operator generates solutions of equation (2.21). Let us illustrate this by an example.

By acting with the operator (3.15) on the function (2.20), we obtain

$$
\begin{aligned}
u_{A}(x, t)= & \widehat{a}\left(x+X_{u}(t)-X_{u_{A}}(t)+M_{3}(t, s)\left(X_{\gamma_{A}}-X_{\gamma}\right), t\right) \times \\
& \times u\left(x+X_{u}(t)-X_{u_{A}}(t)+M_{3}(t, s)\left(X_{\gamma_{A}}-X_{\gamma}\right), t\right) \\
= & \frac{1}{C(t)}\left(C_{0}-\frac{1}{\varepsilon} B_{0}\right)\left(x-X_{u_{A}}(t)+M_{3}(t, s)\left(X_{\gamma_{A}}-X_{\gamma}\right)\right) \\
& \times u\left(x+X_{u}(t)-X_{u_{A}}(t)+M_{3}(t, s)\left(X_{\gamma_{A}}-X_{\gamma}\right), t\right) .
\end{aligned}
$$

The function $u_{A}(x, t)(3.16)$ is a solution of the equation

$$
\left\{-\partial_{t}+\varepsilon \partial_{x}^{2}+\partial_{x} \Lambda x+\varkappa K_{3} X_{u_{A}}(t) \partial_{x}\right\} u_{A}(x, t)=0 .
$$

Notice that the symmetry operators determined by (3.13) are consistent with the operators (3.7) and (3.11) in the one-dimensional case. Moreover, the operator determined by (3.15) corresponds the operator determined by relation (3.7)

$$
\widehat{\widetilde{A}}_{\mathrm{nl}}(\vec{x}, t)=\widehat{A}_{\mathrm{nl}}\left(\vec{x}+\vec{X}_{u}(t), t\right),
$$

which follows from $\dot{M}_{3}(t, s)\left(X_{\gamma_{A}}-X_{\gamma}\right)=-\Lambda M_{3}(t, s)\left(X_{\gamma_{A}}-X_{\gamma}\right)$.

\section{Conclusion}

Symmetry analysis of an equation is usually performed when solutions of the equation are not known, and the basic purpose consists in finding as wide as possible classes of partial solutions by using the symmetries of the equation.

It should be noted that a direct calculation of symmetry operators for a nonlinear equation is, as a rule, difficult because of the complexity of the determining equations [32]. The basic subject for study is the symmetries related to the generators of one-parameter subgroups of a Lie group of symmetry operators [3]. The determining equations for the symmetries are linear, and to solve them, it is necessary to set the structure of the symmetries. Finding of nonlocal symmetries for differential equations or differential symmetries for nonlocal equations from the determining equations, faces mathematical problems.

In this context, the algorithm proposed in this work to calculate the symmetry operators for the FPKE in explicit form is of interest as it provides a possibility to consider the properties of symmetry operators for a nontrivial nonlinear equation. The algorithm is stated in terms of a direct nonlinear evolution operator and the corresponding left-inverse operator, which enables one to vary the structure of the obtained symmetry operators.

As for the considered equation the evolution operator is found, the symmetry operators are not of interest for finding of partial solutions of the equation. However, the symmetry operators can be used to study the properties of the solutions obtained. Furthermore the algebra of symmetry operators of the linear FPKE (2.9) (see, e.g. [33]) can be used to study the symmetry operators of the nonlinear equation (2.2).

The developed approach permits a generalization for FPKE's with a smooth operator symbol of arbitrary form. In this case, we deal with a solution of the FPKE approximate in the small parameter $\varepsilon$ [21]. The FPKE considered in this work arises in constructing the leading term of semiclassical asymptotics for a FPKE with arbitrary coefficients. 


\section{Acknowledgements}

The work was supported by President of the Russian Federation Grant No SS-5103.2006.2, Deutsche Forschungsgemeinschaft. ROR was supported in part by the scholarship of the nonprofit Dynasty Foundation.

\section{References}

[1] Miller W., Jr., Symmetry and separation of variables, Addison-Wesley, London - Amsterdam - Ontario Tokio - New York, 1977.

[2] Malkin M.A., Manko V.I., Dynamic symmetries and coherent states of quantum systems, Nauka, Moscow, 1979 (in Russian).

[3] Ovsjannikov L.V., Group analysis of differential equations, Nauka, Moscow, 1978 (English transl.: Academic Press, New York, 1982).

[4] Anderson R.L., Ibragimov N.H., Lie-Bäcklund transformations in applications, Philadelphia, SIAM, 1979.

[5] Olver P.J., Application of Lie groups to differential equations, Springer, New York, 1986.

[6] Fushchych W.I., Shtelen W.M., Serov N.I., Symmetry analysis and exact solutions of equations of nonlinear mathematical physics, Kluwer, Dordrecht, 1993.

[7] Fushchych W.I., Nikitin A.G., Symmetries of equations of quantum mechanics, Allerton Press Inc., New York, 1994.

[8] Krasilshchik I.S., Lychagin V.V., Vinogradov A.M., Geometry of jet spaces and nonlinear partial differential equations, Advanced Studies in Contemporary Mathematics, Vol. 1, Gordon and Breach Science Publishers, New York, 1986.

[9] Gaeta G., Nonlinear symmetries and nonlinear equations, Mathematics and its Applications, Vol. 299, Kluwer Academic Publishers Group, Dordrecht, 1994.

[10] Frank T.D., Nonlinear Fokker-Planck equations, Springer, Berlin, 2004.

[11] Bellucci S., Trifonov A.Yu., Semiclassically-concentrated solutions for the one-dimensional Fokker-Planck equation with a nonlocal nonlinearity, J. Phys. A: Math. Gen. 38 (2005), L103-L114.

[12] Maslov V.P., The complex WKB method for nonlinear equations. I. Linear theory, Birkhauser Verlag, Basel Boston - Berlin, 1994.

[13] Belov V.V., Dobrokhotov S.Yu., Semiclassical Maslov asymptotics with complex phases. I. General approach, Teor. Mat. Fiz. 92 (1992), 215-254 (English transl.: Theoret. and Math. Phys. 92 (1992), 843-868).

[14] Dobrokhotov S.Yu., Martinez Olive V., Localized asymptotic solutions of the magneto dynamo equation in ABC-fields, Mat. Zametki 54 (1993), no. 4, 45-68 (English transl.: Math. Notes 54 (1993), 1010-1025).

[15] Albeverio S., Dobrokhotov S.Yu., Poteryakhin M., On quasimodes of small diffusion operators corresponding to stable invariant tori with nonregular neighborhoods, Asymptot. Anal. 43 (2005), no. 3, 171-203.

[16] Maslov V.P., Global exponential asymptotic behavior of the solutions of tunnel-type equations and the problem of large deviations, Trudy Mat. Inst. Steklov. 193 (1984), 150-180.

[17] Belov V.V., Shapovalov A.V., Trifonov A.Yu., The trajectory-coherent approximation and the system of moments for the Hartree type equation, Int. J. Math. Math. Sci. 32 (2002), 325-370, math-ph/0012046.

[18] Belov V.V., Trifonov A.Yu., Shapovalov A.V., Semiclassical trajectory-coherent approximations to Hartree type equations, Teor. Mat. Fiz. 130 (2002), 460-492 (English transl.: Theoret. and Math. Phys. 130 (2002), 391-418).

[19] Lisok A.L., Trifonov A.Yu., Shapovalov A.V., The evolution operator of the Hartree-type equation with a quadratic potential, J. Phys. A: Math. Gen. 37 (2004), 4535-4556, math-ph/0312004.

[20] Shapovalov A.V., Trifonov A.Yu., Lisok A.L., Exact solutions and symmetry operators for the nonlocal Gross-Pitaevskii equation with quadratic potential, SIGMA 1 (2005), 007, 14 pages, math-ph/0511010.

[21] Shvedov O.Yu., Semiclasical symmetries Ann. Phys. 296 (2002), 51-89.

[22] Trifonov A.Yu., Trifonova L.B., Fokker-Planck-Kolmogorov equation's with a nonlocal nonlinearity in a semiclassical approximation, Izv. Vyssh. Uchebn. Zaved. Fiz. 45 (2002), no. 2, 121-129 (English transl.: Russian Phys. J. 45 (2002), 118-128). 
[23] Bezverbny A.V., Gogolev A.S., Trifonov A.Yu., Rezaev R.O., Nonlinear Fokker-Planck-Kolmogorov equation in the semiclassical coherent trajectory approximation Izv. Vyssh. Uchebn. Zaved. Fiz. 48 (2005), no. 6, 38-47 (English transl.: Russian Phys. J. 48 (2005), 592-604).

[24] Shiino M., Yoshida K., Chaos-nonchaos phase transitions induced by external noise in ensembles of nonlinearly coupled oscillators, Phys. Rev. E 63 (2001), 026210, 6 pages.

[25] Shiino M., Stability analysis of mean-field-type nonlinear Fokker-Planck equations associated with a generalized entropy and its application to the self-gravitating system, Phys. Rev. E 67 (2003), 056118, 5 pages.

[26] Drozdov A.N., Morillo M., Validity of basic concepts in nonlinear cooperative Fokker-Plank models, Phys. Rev. E 54 (1996), 3304-3313.

[27] Schuller F.P., Vogt P., Product structure of heat phase space and branching Brownian motion, Ann. Phys. 308 (2003), 528-554, math-ph/0209016.

[28] Frank T.D., Classical Langevin equations for the free electron gas and blackbody radiation, J. Phys. A: Math. Gen. 37 (2004), 3561-3567.

[29] Tatarskii V.I., The Wigner representation of quantum mechanics, Uspekhi Fiz. Nauk 139 (1983), 587-619 (English transl.: Soviet Phys. Uspekhi 139 (1983), 311-327).

[30] Popov M.M., Green functions for the Schrödinger equation with a quadric potential, in Problems of Mathematical Physics, Vol. 6, Leningrad, 1973, 119-125 (in Russian).

[31] Dodonov V.V., Malkin I.A., Man'ko V.I., Integrals of motion, Green functions and coherent states of dynamic systems, Internat. J. Theoret. Phys. 14 (1975), 37-54.

[32] Pukhnachov V.V., Transformations of equivalence and the hidden symmetry of evolution equations, Dokl. Akad. Nauk SSSR 294 (1987), 535-538 (English transl.: Sov. Math. Dokl. 34 (1987), 555-558).

[33] Lahno V.I., Spichak S.V., Stogniy V.I., Symmetry analysis of evolution type equations, Computer Research Institute, Moscow - Igevsk, 2004 (in Russian). 